\title{
The Experimental Study of Mine Intrinsically Safe Electromagnet
}

\author{
Li Jing, Zhang Xi and Li Zhi \\ School of Mechanical Electronic \& Information Engineering, China University of Mining \& \\ Technology, Beijing, 100083, China \\ E-mail: m13220189764@163.com
}

Keywords: electro-hydraulic control system; intrinsically safe electromagnet; characteristic test Abstract: Electromagnet, as the actuator of electro hydraulic control system, its performance directly affects the performance of the electro-hydraulic control system. This paper analyses the working principle of intrinsically safe electromagnet, determines the experimental program, and tests the characteristics of the electromagnet.

\section{Introduction}

Intrinsically safe electromagnetic pilot valve is the key components of electro-hydraulic control system. The power requirements are generally less than $1.2 \mathrm{~W}$, which is twentieth of conventional hydraulic valve belonging to low-power solenoid valve. As the motion actuator of electromagnetic pilot valve, the intrinsically safe solenoid plays a vital role. The performance of solenoid directly affects the performance of electromagnetic pilot valve. Therefore, the performance study of intrinsically safe solenoid has a very important significance.

Study on safety technology abroad is relatively mature, the United Kingdom, Germany has formulated and improved safety standards ${ }^{[1]}$. In China, after mounts of domestic and overseas study and investigation on the intrinsically safe type electromagnet, Taiyuan University of technology subject group designs their intrinsically safe type electromagnet and tests its performance ${ }^{[2]}$. A set of static characteristics testing system is developed for the solenoid valve of the solenoid valve of the hydraulic support by Ge Jian from Hebei University of Technology. But the system is unable to complete the test of the dynamic characteristics ${ }^{[3]}$. This paper will continue to study the performance of the electromagnet with the base of the former research group's research result, the development of a set of test system for the characteristics of the solenoid, and to conduct the performance of the electromagnet and parameter test.

\section{The principle of electromagnet test system}

The test system designed in this paper is used for the characteristic test of intrinsically safe solenoid that was made by our research groups and to find out whether it meets the requirements of electromagnetic pilot valve or not. Because there is not a relatively standardized standard for testing the intrinsically safe solenoid ${ }^{[4]}$, therefore, this paper designs a test system for researching the characteristics of the electromagnet, which is based on the reference and the reference of other testing organizations.

Electromagnetic test system is composed of two parts test equipment and computers ${ }^{[5]}$.Experimental device are used for testing data collection. Its components include power supply, optical coupler, acceleration sensor, charge amplifier, filter circuit, voltage amplifier and data acquisition cards, etc. The computer is used for the processing of data which includes synchronization acquisition, performance curve drawing, data storage and display of the results.

Its principle is shown as Fig. 1. 


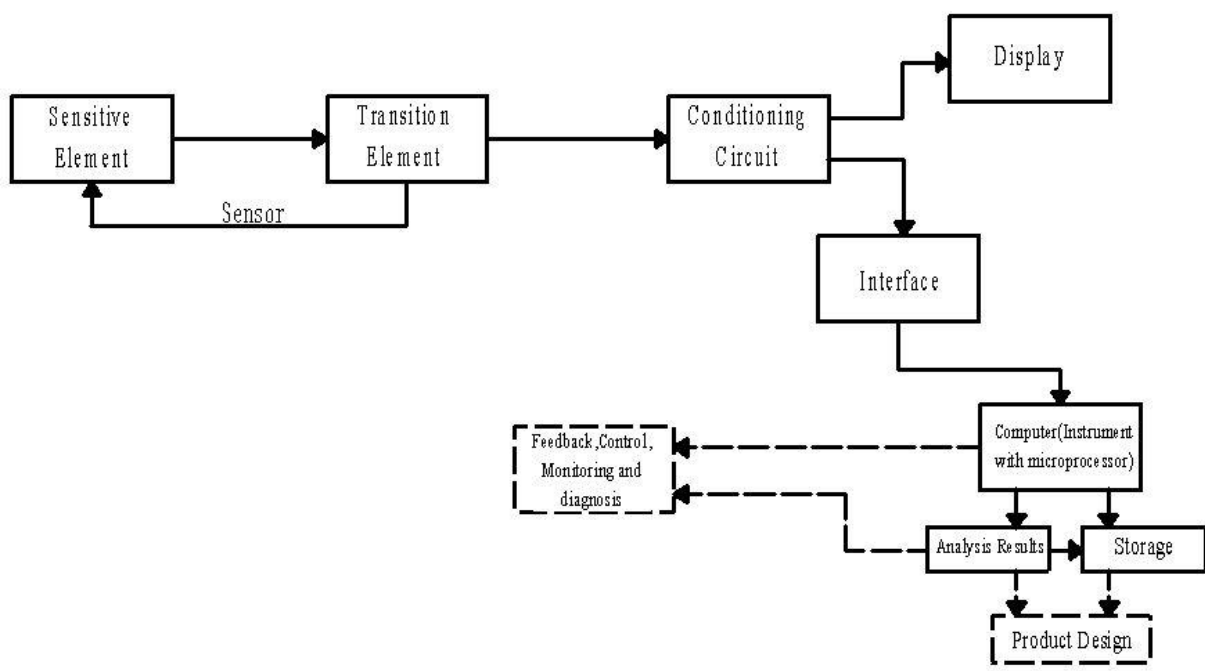

Fig. 1 test system diagram

\section{The design scheme of test system}

The choice of the test scheme of the solenoid performance mainly focuses on how to measure the data and the way to obtain the data. There are two methods to measure the change of the force in the movement of the electromagnet push rod. The first one is to measure the change of the force directly. Secondly, by measuring the change of acceleration in the moving process, the change of the force of electromagnet in the stroke is measured indirectly according to the relationship between acceleration and force.

Because of the necessity to directly measure the changes of electromagnet force, to connect electromagnets and sensors with a power transmission mechanism is required. Because the spring can produce a large deformation under the load, and in the case of small amount of compression, it is not easy to distort. So it is suitable to be the medium of transmission mechanism.

The structure diagram of spring force transmission mechanism of experimental design is shown as Fig. 2 .

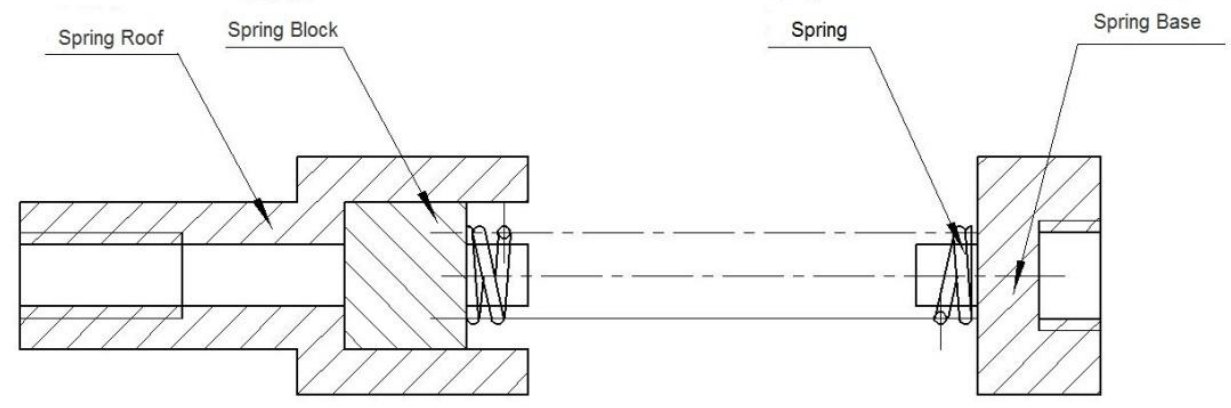

Fig. 2 spring force transmission mechanism

The difficulty of indirect measurement is that the movement time of the electromagnet push rod is short and the displacement is small, so it is the most important problem that how to measure the acceleration of the electromagnet push rod in a very short time.

Direct measurement method is more complex than the indirect measurement method, and it needs to adjust the spring tension level, so that it can reach the level and the pre-tightening force is zero.

Direct measurement requires high processing and operation precision, but its operability is not good. In contrast, the indirect measurement method is simple in mechanical structure, its difficulty lies in the design of the test circuit compared with the direct measurement method. Then, the indirect 
measurement method is more operable, so the method of indirect measurement is used in this experiment.

The diagram of indirect measurement is shown as Fig. 3.

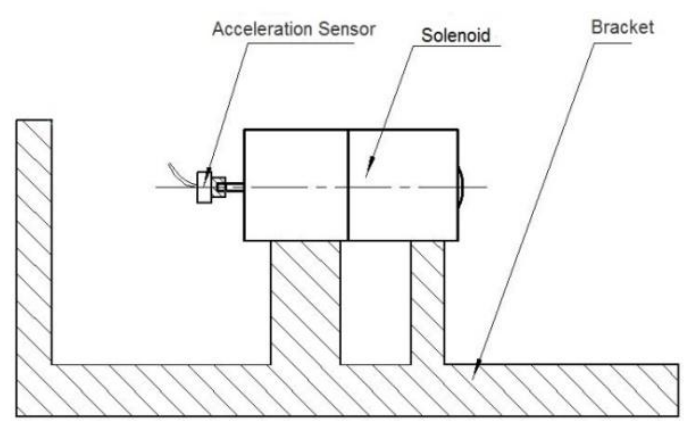

Fig. 3 Indirect measurement organization diagram

\section{The experimental process}

(1) Connect the switching power, an optical coupler, an acceleration sensor, the charge amplifier, filter circuit, a voltage amplifier and a data acquisition card together in turns.

(2) Connect the solenoid with the corresponding synchronous acquisition module

(3) Connect the acceleration sensor through nylon bushings by using 502 glue.

(4) Power on, and the data acquisition card would acquire data, then the PC would process the data further more.

\section{Data analysis}

The acceleration curve of push rod measured by using self-characteristic test system is shown in Fig. 4. Electromagnet handspike do not move in 0.02 seconds, but it is speedy accelerated by overcoming internal resistance after 0.02 seconds. The maximum acceleration of $33 \mathrm{~m} / \mathrm{s}^{2}$ will appears in about 0.15 seconds and the maximum force of the push rod inferred by the relationship between acceleration and force in movement process is about $3 N$.

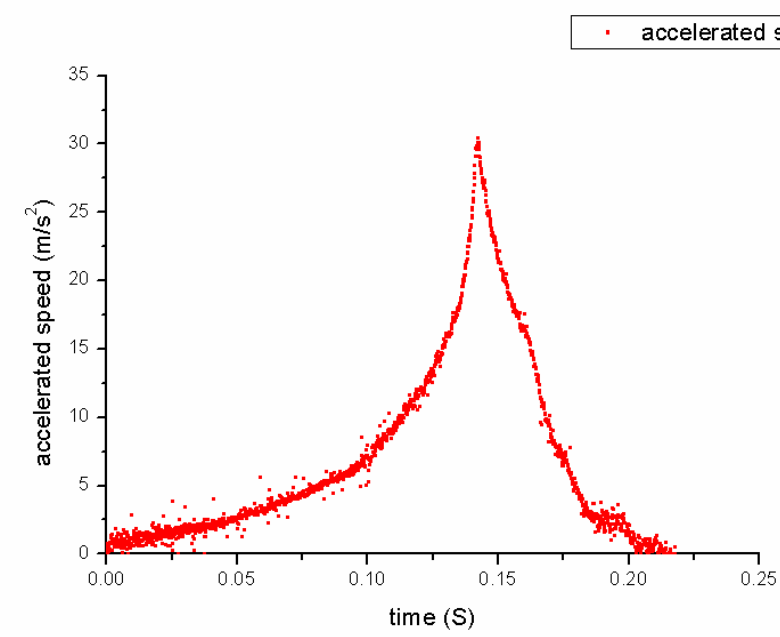

Fig .4 The push rod acceleration curve

\section{Conclusions}

Mine intrinsically safe solenoid is the object of study in this paper. Its working principles are introduced, and the load characteristics are analyzed. The electromagnet's testing system is built by the knowledge of the test technology. The main works of this paper include: 
(1) On the basis of the existing research, the performance testing system based on PCI8326A high speed data acquisition card is set up.

(2) The characteristic test of the machined solenoid has been carried out, and the experimental results are analyzed and studied.

\section{References}

[1] Electrical apparatus for Plosive Gas Atmosphere Part11: Intrinsiesafe "I", Standards Australia International, 2000: 26-29.

[2] Zhang Hong, Lian Zisheng, etc. Numerical Simulation and Experiment of Thrust Characteristic on Electromagnet of Pilot Valve [J]. Journal of China Coal Society, 2008, 33(3), 339-342.

[3] Ge Jian. Static Characteristics Test System of Electromagnet [D]. Tianjin: Hebei University of Technology, 2009.

[4] Wang Junxia. Development of Automatic Test Platform for the Dynamic and Static Performance of Valve [D].Zhejiang: Zhejiang University, 2008, 43-45.

[5] Hu Yanping, Wang Qiang. A Characteristics Test System of Proportional Solenoid [J]. Journal of Hunan University of Science \& Technology (Natural Science Edition), 2012, 27:37-40. 